\title{
Robust Stabilization and Disturbance Rejection of Positive Systems with Time-Varying Delays and Actuator Saturation
}

\author{
Xi Ding, ${ }^{1}$ Zhengrong Xiang, ${ }^{1}$ and Chunyu Yang ${ }^{2}$ \\ ${ }^{1}$ School of Automation, Nanjing University of Science and Technology, Nanjing 210094, China \\ ${ }^{2}$ School of Information and Electrical Engineering, China University of Mining and Technology, Xuzhou 221116, China \\ Correspondence should be addressed to Zhengrong Xiang; xiangzr@mail.njust.edu.cn
}

Received 11 June 2014; Accepted 17 August 2014

Academic Editor: Mohammed Chadli

Copyright (c) $2015 \mathrm{Xi}$ Ding et al. This is an open access article distributed under the Creative Commons Attribution License, which permits unrestricted use, distribution, and reproduction in any medium, provided the original work is properly cited.

\begin{abstract}
This paper focuses on the problems of robust stabilization and disturbance rejection for a class of positive systems with time-varying delays and actuator saturation. First, a convex hull representation is used to describe the saturation characteristics. By constructing an appropriate copositive type Lyapunov functional, we give sufficient conditions for the existence of a state feedback controller such that the closed-loop system is positive and asymptotically stable at the origin of the state space with a domain of attraction. Then, the disturbance rejection performance analysis in the presence of actuator saturation is developed via $L_{1}$-gain. The design method is also extended to investigate the problem of $L_{1}$-gain analysis for uncertain positive systems with time-varying delays and actuator saturation. Finally, three examples are provided to demonstrate the effectiveness of the proposed method.
\end{abstract}

\section{Introduction}

Positive systems, whose states and outputs are nonnegative whenever the initial conditions and inputs are nonnegative, are of fundamental importance to multitudinous applications in areas such as economics, biology, sociology, and communications [1-4]. Recently, positive systems have been investigated by many researchers [5-10]. The linear copositive Lyapunov functional approach has been used to study the stability of positive systems due to the fact that it is less conservative than the traditional quadratic Lyapunov functional method [11]. It is well known that, in real engineering, timedelays are involved in many subjects and fields, such as mechanics, medicine, chemistry, physics, engineering, and control theory [12]. The existence of time-delay may lead to the deterioration of system performance and instability. Many results have been reported for time-delay systems [1318], and a few results on positive systems with time-delay have appeared in [19-21].

On the other hand, in practice, the reaction to exogenous signals is not instantaneous, and the outputs will be inevitably affected. Because of the peculiar nonnegative property of positive systems, it is natural to evaluate the size of such systems via the $L_{1}$-gain in terms of the ratio of input and output signals [19]. Some results on $L_{1}$-gain analysis and control of positive systems have been reported in the literature $[19,22]$.

Recently, several works on positive systems have been done $[12,19,23-28]$. It should be pointed out that, in almost all available results on positive systems, it has been assumed that the actuator provides unlimited amplitude signal. However, actuator saturation is commonly unavoidable in almost all practical control systems because of the existence of physical, technological, or even safety constraints $[29,30]$. Actuator saturation can lead to performance degradation of the closed-loop system; even more, it will make the additional stable closed-loop system unstable for large perturbations. Thus, more and more attention has been focused on the analysis and control synthesis for dynamic systems with actuator saturation for a long time and many methods have been developed to deal with actuator saturation [31-42]. To the best of our knowledge, few results on positive systems with actuator saturation have been proposed [43, 44]. In addition, because of the phenomena of actuator saturation nonlinearities and the peculiar nonnegative property of positive systems, the research of positive systems with actuator 
saturation becomes more difficult for both analysis and synthesis tasks.

In this paper, we focus our attention on the investigation of robust stabilization and disturbance rejection for a class of positive systems with time-varying delays and actuator saturation. The main contributions of this paper lie in three aspects. First, a convex hull representation is used to describe the saturation behavior, and a domain of attraction, which is different from the ellipsoid, is for the first time proposed for positive systems. Secondly, by constructing a copositive type Lyapunov functional, a state-feedback controller design scheme is developed to guarantee the stability with $L_{1}$-gain performance of the resulting closed-loop systems. Thirdly, the proposed controller design method is further extended to the case of uncertain positive systems.

The remainder of this paper is organized as follows. In Section 2, the necessary definitions and lemmas are reviewed. In Section 3, sufficient conditions for the existence of $L_{1}$-gain controller are presented. An extension of the obtained results to uncertain positive systems with time-varying delays and actuator saturation is given in Section 4. Three examples are provided to illustrate the feasibility of the proposed method in Section 5. Concluding remarks are given in Section 6.

Notation. In this paper, $A \succeq 0(\preceq 0)$ means that all the entries of matrix $A$ are nonnegative (nonpositive); $A>0(\prec 0)$ means that all the entries of $A$ are positive (negative); $A \succ B(A \succeq B$ ) means that $A-B>0(A-B \geq 0) ; A^{T}$ means the transpose of matrix $A ; R\left(R^{+}\right)$is the set of all real (positive real) numbers; $R^{n}\left(R_{+}^{n}\right)$ is the $n$-dimensional real (positive) vector space; $R^{n \times k}$ is the set of all real matrices of dimension $(n \times k) ; Z_{+}$refers to the set of all positive integers. For the vector $x \in R^{n}, 1$-norm is denoted by $\|x\|=\sum_{l=1}^{n}\left|x_{l}\right|$, where $x_{l}$ is the $l$ th element of $x$; $1_{n} \in R^{n}$ denotes a column vector with $n$ rows containing only 1 entry; $L_{1}\left[t_{0}, \infty\right)$ is the space of absolute integrable vectorvalued functions on $\left[t_{0}, \infty\right)$; that is, we say $z:\left[t_{0}, \infty\right) \rightarrow R^{k}$ is in $L_{1}\left[t_{0}, \infty\right)$ if $\int_{t_{0}}^{\infty}\|z(t)\| d t<\infty ; H_{\kappa}, \kappa=1,2, \ldots, m$, stands for the $\kappa$ th column of $H_{\kappa}$. For a vector $v \in R_{+}^{n}$ and a positive scalar $\xi, K(v, \xi):=\left\{x \in R_{+}^{n}, x^{T} v \leq \xi\right\}$ denotes a domain of attractive regions, and for a matrix $H \in R^{m \times n}, L(H):=\{x \in$ $\left.R^{n}:\left|H_{\kappa} x\right| \leq 1, \kappa=1,2, \ldots, m\right\}$ denotes a linear region.

\section{Problem Statements and Preliminaries}

Consider the following system with time-varying delays:

$$
\begin{gathered}
\dot{x}(t)=A x(t)+A_{d} x(t-d(t))+E w(t), \\
x\left(t_{0}+\theta\right)=\phi(\theta), \quad \theta \in[-\tau, 0], \\
z(t)=C x(t),
\end{gathered}
$$

where $x(t) \in R^{n}$ is the state vector, $z(t) \in R^{q}$ is the controlled output vector and $w(t) \in R^{p}$ is the disturbance input which belongs to $L_{1}\left[t_{0}, \infty\right), \phi(\theta)$ is the initial condition on $[-\tau, 0]$, $\tau>0, t_{0}$ is the initial time, $A, A_{d}, B, C$, and $E$ are constant matrices of appropriate dimensions, and $d(t)$ denotes the time-varying delay satisfying

$$
0 \leq d(t) \leq \tau, \quad \dot{d}(t) \leq d
$$

where $\tau$ and $d$ are known constants.

Definition 1 (see [24]). System (1) is said to be positive if for any initial condition $\phi(\theta) \succeq 0, \theta \in[-\tau, 0]$, and any inputs $w(t) \geq 0$, it satisfies $x(t) \geq 0$ and $z(t) \geq 0, t \geq t_{0}$.

Definition 2 (see [45]). A is called a Metzler matrix if its offdiagonal entries are nonnegative.

Lemma 3 (see [24]). System (1) is positive if and only if $A$ is a Metzler matrix, and $A_{d} \geq 0, E \geq 0$, and $C \succeq 0$.

Definition 4 (see [19]). For a given positive scalar $\gamma$, system (1) is said to have an $L_{1}$-gain performance level $\gamma$ if the following conditions hold.

(a) System (1) is asymptotically stable when $w(t)=0$.

(b) Under the zero initial condition, that is, $\phi(\theta)=0, \theta \in$ $[-\tau, 0]$, system (1) satisfies

$$
\int_{t_{0}}^{\infty}\|z(t)\| d t \leq \gamma \int_{t_{0}}^{\infty}\|w(t)\| d t, \quad w(t) \neq 0
$$

Now let us consider the following system subject to actuator saturation:

$$
\begin{gathered}
\dot{x}(t)=A x(t)+A_{d} x(t-d(t))+B \text { sat }(u(t))+E w(t), \\
x\left(t_{0}+\theta\right)=\phi(\theta), \quad \theta \in[-\tau, 0], \\
z(t)=C x(t),
\end{gathered}
$$

where $u(t) \in R^{m}$ is the control input vector.

The function $\operatorname{sat}(\cdot): R^{m} \rightarrow R^{m}$ is the saturation function which is defined as

$$
\operatorname{sat}(u)=\left[\begin{array}{llll}
\operatorname{sat}\left(u_{1}\right) & \operatorname{sat}\left(u_{2}\right) & \cdots & \operatorname{sat}\left(u_{m}\right)
\end{array}\right]^{T} \text {, }
$$

where

$$
\operatorname{sat}\left(u_{j}\right)=\operatorname{sgn}\left(u_{j}\right) \min \left\{1,\left|u_{j}\right|\right\}, \quad \forall j=1, \ldots, m .
$$

Let $\Omega$ be the set of all diagonal matrices in $R^{m \times m}$ with diagonal elements that are either 1 or 0 ; then there are $2^{m}$ elements $D_{i}$ in $\Omega$, and for each $i=1,2, \ldots, 2^{m}, D_{i}^{-}=I-D_{i}$ is also an element in $\Omega$.

Lemma 5 (see [46]). Given $F$ and $H$ in $R^{m \times n}$, then

$$
\operatorname{sat}(F x) \in \operatorname{co}\left\{D_{i} F x+D_{i}^{-} H x: i=1,2, \ldots, 2^{m}\right\} \text {, }
$$


for all $x \in R^{n}$ satisfying $\left|H_{\kappa} x\right| \leq 1, \kappa=1,2, \ldots, m$, and $\operatorname{co}\{\cdot\}$ represents the convex hell. Consequently, $\operatorname{sat}(F x)$ can be expressed as

$$
\operatorname{sat}(F x)=\sum_{i=1}^{2^{m}} \eta_{i}\left(D_{i} F+D_{i}^{-} H\right) x
$$

where $\sum_{i=1}^{2^{m}} \eta_{i}=1$ with $0 \leq \eta_{i} \leq 1$.

Consider the following state-feedback control law:

$$
u(t)=F x(t)
$$

where $F$ is a gain matrix to be determined.

From Lemma 5, it is clear that sat $(F x(t))$ satisfies (8) when $x \in L(H)$. Applying controller (9) to system (4) yields the closed-loop system:

$$
\begin{array}{r}
\dot{x}(t)=\sum_{i=1}^{2^{m}} \eta_{i}\left[\left(A+B D_{i} F+B D_{i}^{-} H\right) x(t)\right. \\
\left.+A_{d} x(t-d(t))+E w(t)\right], \\
x\left(t_{0}+\theta\right)=\phi(\theta), \quad \theta \in[-\tau, 0], \\
z(t)=C x(t) .
\end{array}
$$

By Lemma 3, $\sum_{i=1}^{2^{m}} \eta_{i}\left(A+B D_{i} F+B D_{i}^{-} H\right)$ should be Metzler matrices to ensure the positivity of system (10).

The aim of the paper is to determine the controller gain matrix $F$ such that the resulting closed-loop system (10) is positive and asymptotically stable with an $L_{1}$-gain performance.

\section{Main Results}

3.1. Stability Analysis. In this section, we firstly consider the stability of the closed-loop system (10) with $w(t)=0$.

Theorem 6. Given a matrix $H \in R^{m \times n}$, if there exist vectors $v, v \in R_{+}^{n}$, and a matrix $F \in R^{m \times n}$, such that, for $i=$ $1,2, \ldots, 2^{m}$,

$$
\begin{gathered}
A+B D_{i} F+B D_{i}^{-} H \text { are Metzler matrices, } \\
\left(A+B D_{i} F+B D_{i}^{-} H\right)^{T} v+v \prec 0 \\
A_{d}^{T} v-(1-d) v \prec 0 \\
v \geq\left|H_{\kappa}\right|^{T}, \quad \kappa=1,2, \ldots, m
\end{gathered}
$$

then the closed-loop system (10) with $w(t)=0$ is positive and asymptotically stable for any initial states satisfying

$$
\begin{array}{r}
\Gamma(\phi)=(1+\tau)\left(\max _{r \in \underline{n}} v_{r}+\max _{r \in \underline{n}} v_{r}\right) \sup _{\theta \in[-\tau, 0]}|\phi(\theta)| \leq 1, \\
\underline{n}=\{1,2, \ldots, n\},
\end{array}
$$

where $v_{r}\left(v_{r}\right)$ is the rth element of $v(v)$.

Proof. For any $x(t) \in K(v, 1)$, that is, for any $x(t) \in R_{n}^{+}$ satisfying $x^{T}(t) v \leq 1$, we have from (14) that $\left|H_{\kappa} x(t)\right| \leq 1$, $\kappa=1,2, \ldots, m$, that is, $x \in L(H)$; therefore, $K(v, 1) \subset L(H)$. When $w(t)=0$, system (10) can be written by the following representation:

$$
\begin{gathered}
\dot{x}(t)=\sum_{i=1}^{2^{m}} \eta_{i}\left[\left(A+B D_{i} F+B D_{i}^{-} H\right) x(t)\right. \\
\left.+A_{d} x(t-d(t))\right], \\
x\left(t_{0}+\theta\right)=\phi(\theta), \quad \theta \in[-\tau, 0], \\
z(t)=C x(t) .
\end{gathered}
$$

By Lemma 3, it is easy to get from (11) that system (10) is positive. Choose the following copositive type Lyapunov functional candidate for system (10):

$$
V(t)=V_{1}(t)+V_{2}(t)
$$

where

$$
V_{1}(t)=x^{T}(t) v
$$

$$
V_{2}(t)=\int_{t-d(t)}^{t} x^{T}(s) v d s
$$

and $v, v$ are positive vectors to be determined.

When $w(t)=0$, along the trajectory of system (10), we have, $\forall x(t) \in K(v, 1)$,

$$
\begin{gathered}
\dot{V}_{1}(t)=\sum_{i=1}^{2^{m}} \eta_{i}\left[x^{T}(t)\left(A+B D_{i} F+B D_{i}^{-} H\right)^{T} v\right. \\
\left.+x^{T}(t-d(t)) A_{d}^{T} v\right], \\
\dot{V}_{2}(t)=x^{T}(t) v-(1-\dot{d}(t)) x^{T}(t-d(t)) v \\
\leq x^{T}(t) v-(1-d) x^{T}(t-d(t)) v .
\end{gathered}
$$


It follows that

$$
\begin{array}{r}
\dot{V}(t) \leq \sum_{i=1}^{2^{m}} \eta_{i}\left\{x^{T}(t)\left[\left(A+B D_{i} F+B D_{i}^{-} H\right)^{T} v+v\right]\right. \\
\left.+x^{T}(t-d(t))\left(A_{d}^{T} v-(1-d) v\right)\right\}, \\
\forall x(t) \in K(v, 1) .
\end{array}
$$

Combining (12)-(14), we obtain

$$
\dot{V}(t)<0, \quad \forall x(t) \in K(v, 1) .
$$

Therefore, system (10) with $w(t)=0$ is locally asymptotically stable.

Furthermore, it can be obtained from (17) that

$$
\begin{gathered}
x^{T}(t) v \leq V(t) \\
V\left(t_{0}\right)=x^{T}\left(t_{0}\right) v+\int_{t_{0}-d\left(t_{0}\right)}^{t_{0}} x^{T}(s) v d s \\
\leq x^{T}\left(t_{0}\right) v+\tau \sup _{\theta \in[-\tau, 0]} \phi^{T}(\theta) v \\
\leq(1+\tau)\left(\max _{r \in \underline{n}} v_{r}+\max _{r \in \underline{n}} v_{r}\right) \sup _{\theta \in[-\tau, 0]}|\phi(\theta)| \\
=\Gamma(\phi) .
\end{gathered}
$$

Therefore, all the trajectories of $x(t)$ that start from $\Gamma(\phi) \leq$ 1 will remain inside of $K(v, 1)$.

The proof is completed.

3.2. $L_{1}$-Gain Analysis. The following theorem establishes a condition under which the closed-loop system (10) possesses positivity and has an $L_{1}$-gain performance.

Theorem 7. Given positive constants $\gamma, w_{m}$, and a matrix $H \in$ $R^{m \times n}$, if there exist $v, v \in R_{+}^{n}$, and a matrix $F \in R^{m \times n}$, such that, for $i=1,2, \ldots, 2^{m},(11),(13)$, and the following conditions hold,

$$
\begin{gathered}
\left(A+B D_{i} F+B D_{i}^{-} H\right)^{T} v+v+C^{T} 1_{q} \prec 0, \\
E^{T} v-\gamma I_{p} \prec 0, \\
v \geq\left(1+\gamma w_{m}\right)\left|H_{\kappa}\right|^{T}, \quad \kappa=1,2, \ldots, m,
\end{gathered}
$$

then the closed-loop system (10) is positive and asymptotically stable with an $L_{1}$-gain performance level $\gamma$ for any initial states satisfying (15).
Proof. By Lemma 3, it is easy to get from (11) that system (10) is positive. Choose the Lyapunov functional candidate (17). By Theorem 6, the stability of system (10) with $w(t)=0$ is ensured if (13), (23)-(25) hold. To establish the $L_{1}$-gain performance, we define

$$
J_{N}=\int_{t_{0}}^{\infty}(\|z(t)\|-\gamma\|w(t)\|) d t
$$

Under zero initial condition, we have

$$
\begin{aligned}
J_{N}= & \int_{t_{0}}^{\infty}(\|z(t)\|-\gamma\|w(t)\|+\dot{V}(t)) d t \\
& +\left.V(t)\right|_{t=t_{0}}-\left.V(t)\right|_{t=\infty} \\
\leq & \int_{t_{0}}^{\infty}(\|z(t)\|-\gamma\|w(t)\|+\dot{V}(t)) d t .
\end{aligned}
$$

From (13), (23)-(25), we obtain

$$
\|z(t)\|-\gamma\|w(t)\|+\dot{V}(t)<0 .
$$

Under zero initial condition, it gives rise to

$$
\int_{t_{0}}^{\infty}\|z(t)\| d t<\gamma \int_{t_{0}}^{\infty}\|w(t)\| d t
$$

Considering that $w(t) \in L_{1}\left[t_{0}, \infty\right)$, there exists a constant $w_{m}<\infty$ satisfying

$$
\int_{t_{0}}^{\infty}\|w(t)\| d t \leq w_{m} .
$$

Then from (28) and (30) we can get

$$
x^{T}(t) v \leq V(t) \leq V\left(t_{0}\right)+\gamma w_{m} .
$$

It means that if $\Gamma(\phi) \leq 1$, that is, $V\left(t_{0}\right) \leq 1$, then $V(t) \leq$ $1+\gamma w_{m}$ and hence $x(t) \in K\left(v, 1+\gamma w_{m}\right)$.

Therefore, system (10) has an $L_{1}$-gain performance level $\gamma$, and all trajectories will remain inside of $K\left(\nu, 1+\gamma w_{m}\right)$.

The proof is completed.

In what follows, we will give a method for the controller design based on Theorem 7 .

From the definition of the Metzler matrix, (11) can be converted into

$$
e_{g}\left(A+B D_{i} F+B D_{i}^{-} H\right) e_{l}^{T} \geq 0, \quad \forall g, l=1,2, \ldots n, g \neq l,
$$

where

$$
e_{g}=\left[\begin{array}{ccc}
\overbrace{0 \cdots 0}^{g-1} & 1 & \overbrace{0 \cdots 0}^{n-g}
\end{array}\right], \quad e_{l}=\left[\begin{array}{lll}
\overbrace{0 \cdots 0}^{l-1} & 1 & \overbrace{0 \cdots 0}^{n-l}
\end{array}\right] .
$$


Remark 8. It should be noted that both $F$ and $v$ are variables to be determined in Theorem 7, and we cannot directly compute $F$ by using the LMI (linear matrix inequality) method. In order to obtain $F$, we introduce vectors $g_{i}$ satisfying $g_{i} \geq$ $F^{T} D_{i}^{T} B^{T} v, i=1,2, \ldots, 2^{m}$; then (23) holds if the following inequality is satisfied:

$$
\left(A+B D_{i}^{-} H\right)^{T} v+g_{i}+v+C^{T} 1_{q} \prec 0 .
$$

Thus, we can firstly obtain $v, v$, and $g_{i}$ by solving (13), (24)-(25), and (34). Then from (32) and $g_{i} \geq F^{T} D_{i}^{T} B^{T} v$, $i=1,2, \ldots, 2^{m}$, we can get $F$.

Remark 9. There are several results on the stabilization of positive systems with time-varying delays [19, 22, 26]; however, the controllers proposed in these papers may fail to work when the actuator is subject to saturation. In this paper, the actuator saturation, which brings difficulties for the controller design, is taken into account, and the convex hull technique is used to deal with it. The controller proposed in Theorem 7 can guarantee the positivity and the $L_{1}$-gain performance of the closed-loop system despite the existence of actuator saturation.

\section{Extension to Uncertain Case}

In this section, we will extend the results proposed in previous section to the following uncertain positive system:

$$
\begin{gathered}
\dot{x}(t)=\widehat{A} x(t)+\widehat{A}_{d} x(t-d(t))+E w(t), \\
x\left(t_{0}+\theta\right)=\phi(\theta), \quad \theta \in[-\tau, 0], \\
z(t)=C x(t),
\end{gathered}
$$

where $x(t) \in R_{+}^{n}$ is the state vector, $z(t) \in R_{+}^{q}$ is the controlled output vector, $w(t) \in R_{+}^{p}$ is the disturbance input which belongs to $L_{1}[0, \infty), \phi(\theta)$ is the initial condition on $[-\tau, 0]$, $\tau>0$, and $d(t)$ denotes the time-varying delay satisfying (2).

$\widehat{A}$ and $\widehat{A}_{d}$ are uncertain matrices satisfying

$$
\underline{A} \preceq \widehat{A} \preceq \bar{A}, \quad \underline{A}_{d} \preceq \widehat{A}_{d} \preceq \bar{A}_{d},
$$

where $\underline{A}, \bar{A}, \underline{A}_{d}$, and $\bar{A}_{d}$ are known constant matrices.

Lemma 10. If $\underline{A}$ is a Metzler matrix and $\underline{A}_{d} \geq 0, C \succeq 0$, and $E \geq 0$, then system (35) is positive.

Proof. Because $\underline{A}$ is a Metzler matrix and $\underline{A}_{d} \geq 0, C \succeq$ 0 , and $E \geq 0$, it is easy to obtain that $\widehat{A}$ is a Metzler matrix and $\widehat{A}_{d} \geq 0$; then system (35) is positive.

The proof is completed.
Consider the following system with actuator saturation:

$$
\begin{gathered}
\dot{x}(t)=\widehat{A} x(t)+\widehat{A}_{d} x(t-d(t))+B \text { sat }(u(t))+E w(t), \\
x\left(t_{0}+\theta\right)=\phi(\theta), \quad \theta \in[-\tau, 0], \\
z(t)=C x(t),
\end{gathered}
$$

where $u(t) \in R_{+}^{m}$ is the control input vector.

Similarly, the system (37) can be rewritten as the following closed-loop system:

$$
\begin{array}{r}
\dot{x}(t)=\sum_{i=1}^{2^{m}} \eta_{i}\left[\left(\widehat{A}+B D_{i} F+B D_{i}^{-} H\right) x(t)\right. \\
\left.+\widehat{A}_{d} x(t-d(t))+E w(t)\right], \\
x\left(t_{0}+\theta\right)=\phi(\theta), \quad \theta \in[-\tau, 0], \\
z(t)=C x(t) .
\end{array}
$$

By Lemma 10, $\sum_{i=1}^{2^{m}} \eta_{i}\left(\underline{A}+B D_{i} F+B D_{i}^{-} H\right)$ should be Metzler matrices to ensure the positivity of system (38).

The following theorem gives sufficient conditions which ensure the positivity and $L_{1}$-gain property of the closed-loop system (38).

Theorem 11. Given positive constants $\gamma, w_{m}$, and a matrix $H \in R^{m \times n}$, if there exist $v, v \in R_{+}^{n}$, and a matrix $F \in R^{m \times n}$, such that, for $i=1,2, \ldots, 2^{m},(24)-(25)$ and the following conditions hold,

$$
\begin{gathered}
\underline{A}+B D_{i} F+B D_{i}^{-} H \text { are Metzler matrices } \\
\left(\bar{A}+B D_{i} F+B D_{i}^{-} H\right)^{T} v+v+C^{T} 1_{q} \prec 0 \\
\bar{A}_{d}^{T} v-(1-d) v \prec 0
\end{gathered}
$$

then the closed-loop system (38) is positive and asymptotically stable with an $L_{1}$-gain performance level $\gamma$ for any initial states satisfying (15).

Proof. By Theorem 7, it is easy to get from (14) and (39) that $K\left(v, 1+\gamma w_{m}\right) \subset L(H)$ and system (38) is positive.

Choose the Lyapunov functional candidate (17). Along the trajectory of system (38), we have

$$
\begin{array}{r}
\dot{V}(t) \leq \sum_{i=1}^{2^{m}} \eta_{i}\left\{x^{T}(t)\left[\left(\bar{A}+B D_{i} F+B D_{i}^{-} H\right)^{T} v+v+C^{T} 1_{q}\right]\right. \\
+x^{T}(t-d(t))\left(\bar{A}_{d}^{T} v-(1-d) v\right) \\
\left.+w^{T}(t)\left(E^{T} v-\gamma I_{p}\right)\right\}, \\
\forall x(t) \in K\left(v, 1+\gamma w_{m}\right) .
\end{array}
$$


When $w(t)=0$, we obtain

$$
\dot{V}(t)<0, \quad \forall x(t) \in K\left(v, 1+\gamma w_{m}\right) .
$$

Therefore, system (38) with $w(t)=0$ is locally asymptotically stable.

When $w(t) \neq 0$, similar to the proof line of Theorem 7 , the $L_{1}$-gain performance can be obtained.

The proof is completed.

From the definition of the Metzler matrix, (39) can be converted into

$$
e_{g}\left(\underline{A}+B D_{i} F+B D_{i}^{-} H\right) e_{l}^{T} \geq 0, \quad \forall g, l=1,2, \ldots n, g \neq l,
$$

where

$$
e_{g}=\left[\begin{array}{lll}
\overbrace{0 \cdots 0}^{g-1} & 1 & \stackrel{n-g}{0 \cdots 0}
\end{array}\right], \quad e_{l}=\left[\begin{array}{ccc}
\frac{l-1}{0 \cdots 0} & 1 & \stackrel{n-l}{0 \cdots 0}
\end{array}\right] .
$$

Remark 12. It should be noted that condition (40) is not expressed in the form of LMI. We can adopt the method proposed in Remark 8 to find the gain matrix $F$; that is to say, we can firstly get $v, v$, and $g_{i}$ by solving (13), (24)-(25), and

$$
\left(\bar{A}+B D_{i}^{-} H\right)^{T} v+g_{i}+v+C^{T} 1_{q} \prec 0 .
$$

Then from (44) and $g_{i} \geq F^{T} D_{i}^{T} B^{T} v, i=1,2, \ldots, 2^{m}$, we can obtain the gain matrix $F$.

\section{Examples}

In this section, three examples are presented to check the validity of the proposed results.

Example 1. Consider system (4) with the following parameters:

$$
\begin{gathered}
A=\left[\begin{array}{cc}
-1.5 & 2 \\
2.5 & -2
\end{array}\right], \quad A_{d}=\left[\begin{array}{cc}
0.02 & 0.012 \\
0 & 0.03
\end{array}\right], \\
B=\left[\begin{array}{cc}
0.31 & 0.42 \\
0.22 & 0.1
\end{array}\right], \quad C=\left[\begin{array}{cc}
0.33 & 0.12 \\
0.13 & 0.2
\end{array}\right], \quad E=\left[\begin{array}{c}
0.35 \\
0.4
\end{array}\right] .
\end{gathered}
$$

Let $\tau=0.1, d=0.2, \gamma=1.9, w_{m}=0.1$, and

$$
\begin{gathered}
H=\left[\begin{array}{rr}
-1.4 & -0.5 \\
-0.9 & -1.2
\end{array}\right], \quad D_{1}=\left[\begin{array}{ll}
0 & 0 \\
0 & 0
\end{array}\right], \quad D_{2}=\left[\begin{array}{ll}
0 & 0 \\
0 & 1
\end{array}\right], \\
D_{3}=\left[\begin{array}{ll}
1 & 0 \\
0 & 0
\end{array}\right], \quad D_{4}=\left[\begin{array}{ll}
1 & 0 \\
0 & 1
\end{array}\right] .
\end{gathered}
$$

Solving the matrix inequalities (13), (24)-(25), and (34) in Theorem 7 gives rise to

$$
\begin{array}{rlrl}
v & =\left[\begin{array}{l}
2.2811 \\
1.9938
\end{array}\right], & v=\left[\begin{array}{l}
0.8324 \\
0.6800
\end{array}\right], \\
g_{1}=\left[\begin{array}{l}
0.4790 \\
0.1886
\end{array}\right], & g_{2}=\left[\begin{array}{l}
-1.9882 \\
-1.7587
\end{array}\right], \\
g_{3}=\left[\begin{array}{l}
-1.3932 \\
-0.9427
\end{array}\right], & g_{4}=\left[\begin{array}{l}
-3.5923 \\
-2.3316
\end{array}\right] .
\end{array}
$$

Then from (32) and $g_{i} \geq F^{T} D_{i}^{T} B^{T} v, i=1,2, \ldots, 2^{m}$, we can get

$$
F=\left[\begin{array}{ll}
-2.2708 & -1.6108 \\
-2.7555 & -2.1416
\end{array}\right]
$$

and $A+B D_{i} F+B D_{i}^{-} H, i=1,2, \ldots, 2^{m}$, are Metzler matrices.

The simulation results are shown in Figures 1-3, where the initial condition of the systems is $x(0)=$ $\left[\begin{array}{ll}0.19 & 0.28\end{array}\right]^{T}, x(\theta)=\left[\begin{array}{ll}0 & 0\end{array}\right]^{T}, \theta=[-0.1,0)$, and the disturbance input is $w(t)=0.05 e^{-0.5 t}$. Figure 1 shows the domain of attraction. Figure 2 plots the state responses of the closedloop system. Figure 3 shows the control signals $u(t)$ and $\operatorname{sat}(u(t))$. It is not hard to find that the feedback controller can guarantee the positivity and the asymptotical stability of the closed-loop system.

Example 2. Consider system (37) with the following parameters:

$$
\begin{gathered}
\underline{A}=\left[\begin{array}{cc}
-2 & 3 \\
3 & -3
\end{array}\right], \quad \bar{A}=\left[\begin{array}{cc}
-1 & 2 \\
2 & -2
\end{array}\right], \\
\underline{A}_{d}=\left[\begin{array}{cc}
0.01 & 0.005 \\
0 & 0.02
\end{array}\right], \quad \bar{A}_{d}=\left[\begin{array}{cc}
0.02 & 0.01 \\
0 & 0.03
\end{array}\right], \\
B=\left[\begin{array}{cc}
0.31 & 0.42 \\
0.22 & 0.1
\end{array}\right], \quad C=\left[\begin{array}{ll}
0.5 & 0.1 \\
0.1 & 0.2
\end{array}\right], \quad E=\left[\begin{array}{l}
0.3 \\
0.4
\end{array}\right] .
\end{gathered}
$$

Let $\tau=0.1, d=0.2, \gamma=2.87, w_{m}=0.1$, and

$$
\begin{gathered}
H=\left[\begin{array}{cc}
-1.2 & -0.4 \\
-0.8 & -1
\end{array}\right], \quad D_{1}=\left[\begin{array}{ll}
0 & 0 \\
0 & 0
\end{array}\right], \\
D_{2}=\left[\begin{array}{ll}
0 & 0 \\
0 & 1
\end{array}\right], \quad D_{3}=\left[\begin{array}{ll}
1 & 0 \\
0 & 0
\end{array}\right], \quad D_{4}=\left[\begin{array}{ll}
1 & 0 \\
0 & 1
\end{array}\right] .
\end{gathered}
$$




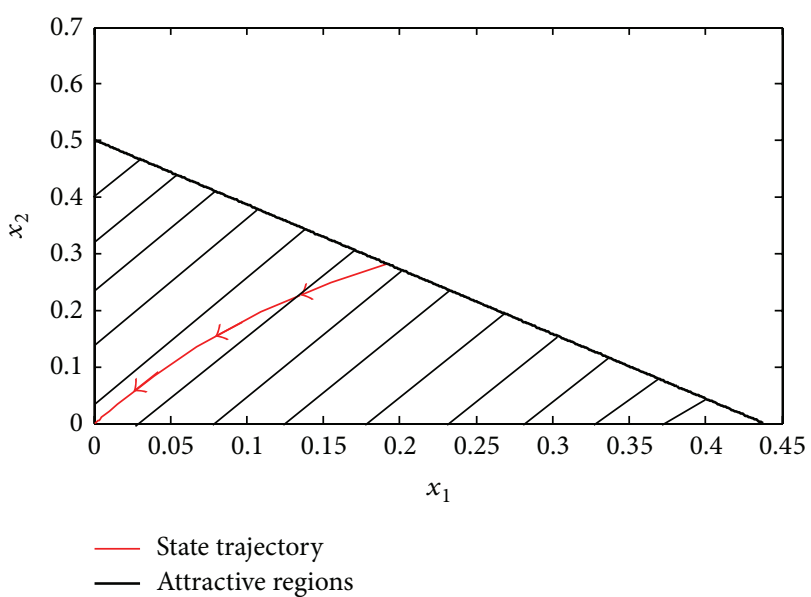

Figure 1: Attractive regions and state trajectory in Example 1.

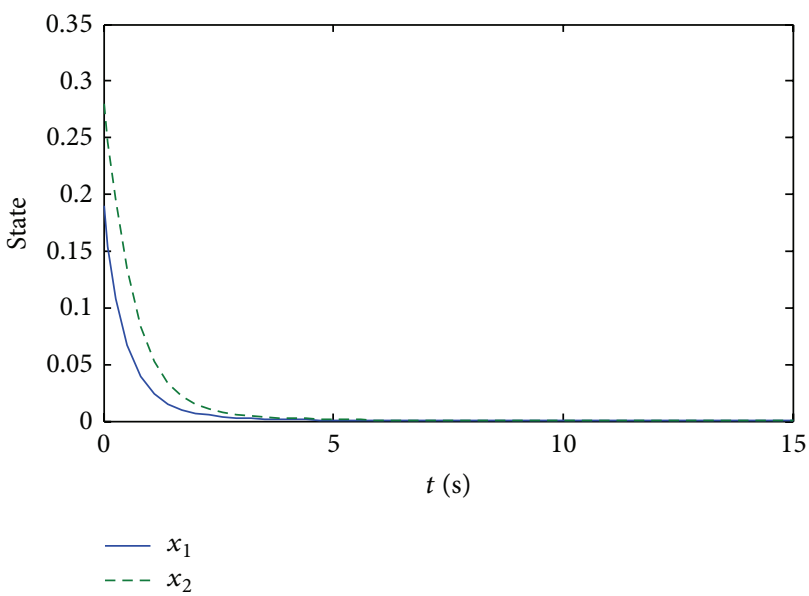

FIGURE 2: State responses of the closed-loop system in Example 1.

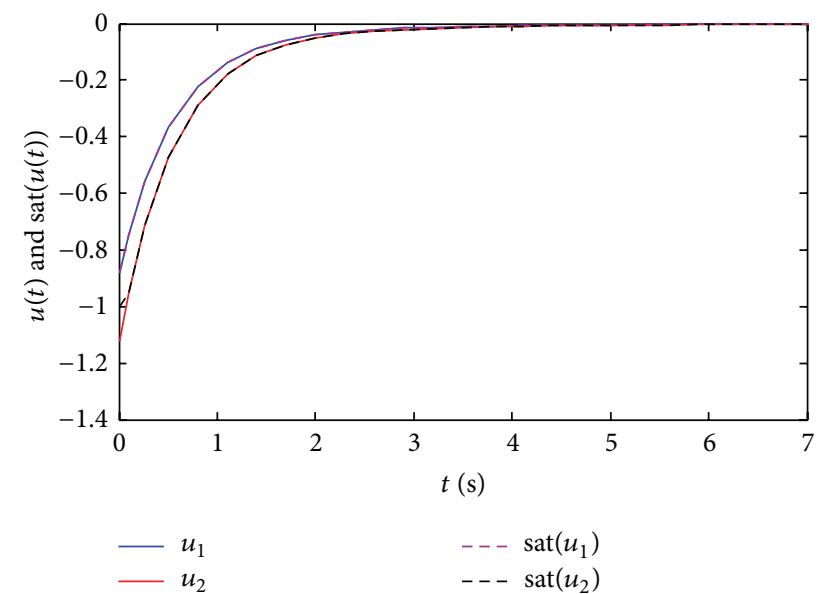

FIGURE 3: $u(t)$ and sat $(u(t))$ of the closed-loop system in Example 1.

Solving the matrix inequalities (13), (24)-(25), and (46) in Theorem 11 gives rise to

$$
v=\left[\begin{array}{l}
3.7490 \\
3.0111
\end{array}\right], \quad v=\left[\begin{array}{l}
0.5552 \\
0.5930
\end{array}\right],
$$

$$
\begin{array}{ll}
g_{1}=\left[\begin{array}{l}
0.1308 \\
0.1184
\end{array}\right], & g_{2}=\left[\begin{array}{l}
-6.1343 \\
-6.5343
\end{array}\right], \\
g_{3}=\left[\begin{array}{l}
-6.8233 \\
-5.3885
\end{array}\right], & g_{4}=\left[\begin{array}{l}
-8.3239 \\
-7.2642
\end{array}\right] .
\end{array}
$$

Then from (44) and $g_{i} \geq F^{T} D_{i}^{T} B^{T} v, i=1,2, \ldots, 2^{m}$, we can get

$$
F=\left[\begin{array}{ll}
-4.0921 & -3.3218 \\
-3.7483 & -3.6654
\end{array}\right]
$$

and $A+B D_{i} F+B D_{i}^{-} H, i=1,2, \ldots, 2^{m}$, are Metzler matrices.

The simulation results are shown in Figures 4-6, where the initial condition of the systems is $x(0)=\left[\begin{array}{ll}0.25 & 0.01\end{array}\right]^{T}$, $x(\theta)=\left[\begin{array}{ll}0 & 0\end{array}\right]^{T}, \theta=[-0.1,0)$ and the disturbance input is $w(t)=0.05 e^{-0.5 t}$. Figure 4 plots the domain of attraction. Figure 5 shows the state responses of the closed-loop system. Figure 6 shows the control signals $u(t)$ and sat $(u(t))$. It can be seen from Figures $4-6$ that the closed-loop system is positive and asymptotically stable. This demonstrates the effectiveness of the proposed approach.

Example 3. Consider a model of virus treatment which can be described as system (4) [6]. The system parameters are as follows:

$$
A=\left[\begin{array}{cc}
-1.6 & 1 \\
1.59 & -1
\end{array}\right], \quad A_{d}=\left[\begin{array}{cc}
0.02 & 0.012 \\
0 & 0.03
\end{array}\right],
$$

$$
B=\left[\begin{array}{cc}
0.1 & 0.42 \\
0.21 & 0.1
\end{array}\right], \quad C=\left[\begin{array}{cc}
0.33 & 0.12 \\
0.13 & 0.2
\end{array}\right], \quad E=\left[\begin{array}{c}
0.35 \\
0.4
\end{array}\right]
$$

where $x_{1}(t)$ and $x_{2}(t)$ are virus populations of two different viral genotypes.

$$
\begin{aligned}
& \text { Let } \tau=0.1, d=0.2, \gamma=1.2, w_{m}=0.1 \text {, and } \\
& H=\left[\begin{array}{rr}
-1.4 & -0.5 \\
-0.9 & -1.2
\end{array}\right], \quad D_{1}=\left[\begin{array}{ll}
0 & 0 \\
0 & 0
\end{array}\right], \\
& D_{2}=\left[\begin{array}{ll}
0 & 0 \\
0 & 1
\end{array}\right], \quad D_{3}=\left[\begin{array}{ll}
1 & 0 \\
0 & 0
\end{array}\right], \quad D_{4}=\left[\begin{array}{ll}
1 & 0 \\
0 & 1
\end{array}\right] .
\end{aligned}
$$

Solving the matrix inequalities (13), (24)-(25), and (32)(34) in Theorem 7 gives rise to

$$
F=\left[\begin{array}{rr}
-2.6601 & -2.0812 \\
-1.5620 & -1.8498
\end{array}\right] \text {. }
$$

Choosing the initial condition $x(0)=\left[\begin{array}{ll}0.2 & 0.4\end{array}\right]^{T}, x(\theta)=$ $\left[\begin{array}{ll}0 & 0\end{array}\right]^{T}, \theta=[-0.1,0)$ and the disturbance input $w(t)=$ $0.05 e^{-0.5 t}$, the simulation results are shown in Figures 7 and 8 . 


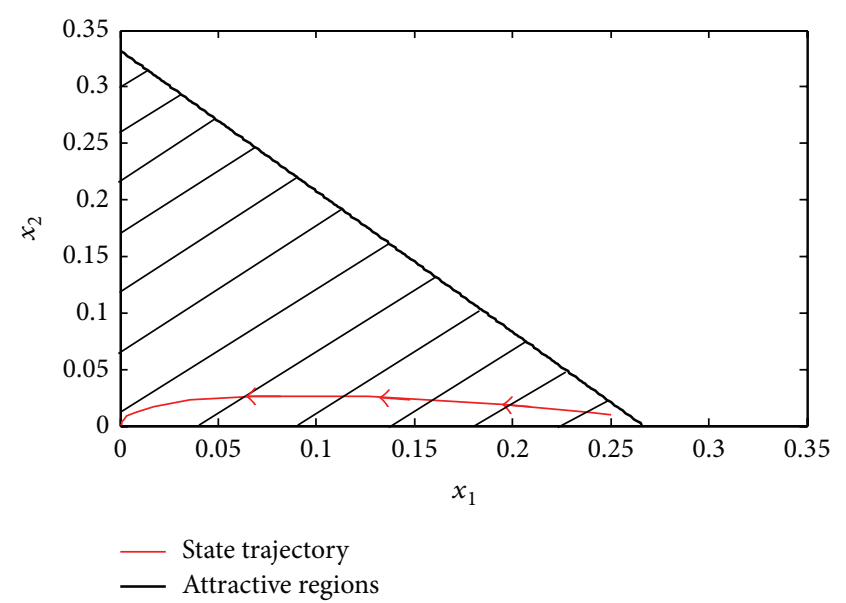

FIgURE 4: Attractive regions and state trajectory in Example 2.

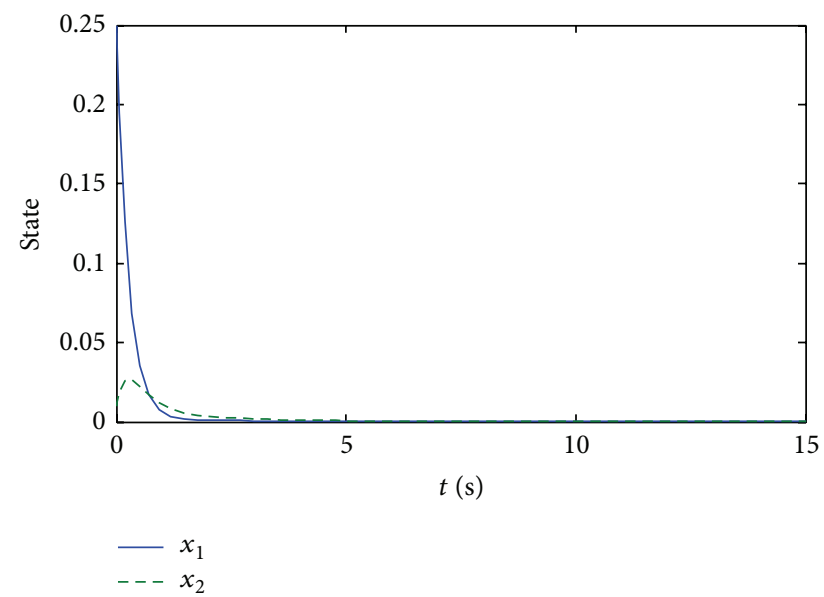

FIgURE 5: State responses of the closed-loop system in Example 2.

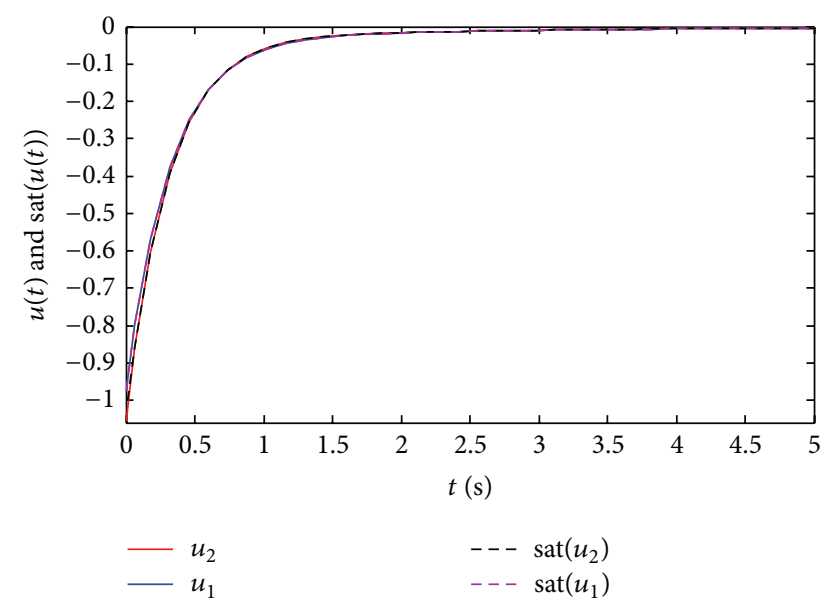

Figure 6: $u(t)$ and sat $(u(t))$ of the closed-loop system in Example 2.

\section{Conclusions}

In this paper, we have investigated the problems of robust stabilization and disturbance rejection for a class of positive systems with time-varying delays and actuator saturation. An appropriate copositive type Lyapunov functional is employed

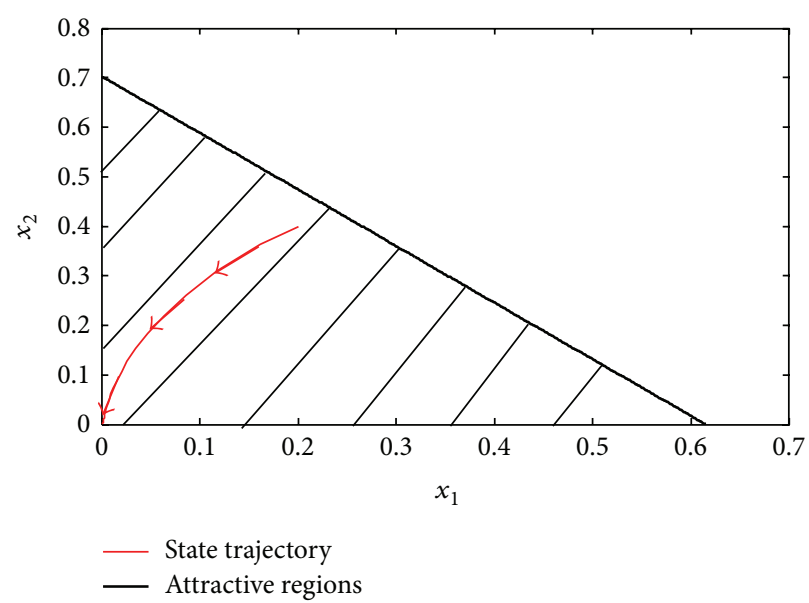

FIgURE 7: Attractive regions and state trajectory in Example 3.

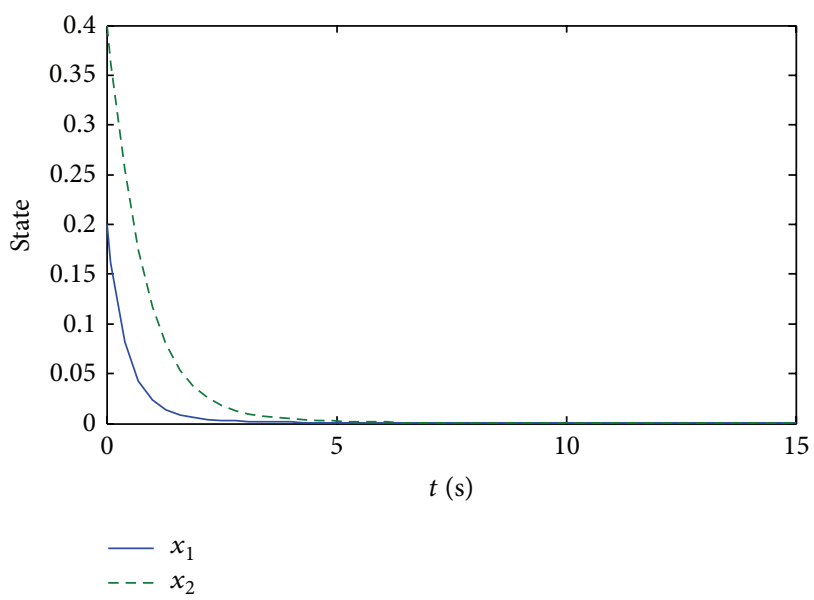

FIGURE 8: State responses of the closed-loop system in Example 3.

to ensure the stability and $L_{1}$-gain performance of the positive systems. Both the existence conditions and the explicit characterization of the desired controller are derived in terms of LMIs. Finally, three examples are provided to illustrate the validity of the theoretical results.

\section{Conflict of Interests}

The authors declare that there is no conflict of interests regarding the publication of this paper.

\section{Acknowledgments}

This work was supported by the National Natural Science Foundation of China under Grant no. 61273120 and the Postgraduate Innovation Project of Jiangsu Province (Grant no. CXZZ13_0208).

\section{References}

[1] L. Gurvits, R. Shorten, and O. Mason, "On the stability of switched positive linear systems," IEEE Transactions on Automatic Control, vol. 52, no. 6, pp. 1099-1103, 2007. 
[2] R. Shorten, D. Leith, J. Foy, and R. Kilduff, “Towards an analysis and design framework for congestion control in communication networks," in Proceeding of the 12th Yale Workshop on Adaptive and Learning Systems, 2003.

[3] R. Shorten, F. Wirth, and D. Leith, "A positive systems model of TCP-like congestion control: asymptotic results," IEEE/ACM Transactions on Networking, vol. 14, no. 3, pp. 616-629, 2006.

[4] A. Jadbabaie, J. Lin, and A. S. Morse, "Coordination of groups of mobile autonomous agents using nearest neighbor rules," IEEE Transactions on Automatic Control, vol. 48, no. 6, pp. 988-1001, 2003.

[5] M. Bolajraf, F. Tadeo, T. Alvarez, and M. A. Rami, "State-feedback with memory for controlled positivity with application to congestion control," IET Control Theory \& Applications, vol. 4, no. 10, pp. 2041-2048, 2010.

[6] E. Hernandez-Vargas, P. Colaneri, R. Middleton, and F. Blanchini, "Discrete-time control for switched positive systems with application to mitigating viral escape," International Journal of Robust and Nonlinear Control, vol. 21, no. 10, pp. 1093-1111, 2011.

[7] T. Kaczorek, "The choice of the forms of Lyapunov functions for a positive 2D Roesser model," International Journal of Applied Mathematics and Computer Science, vol. 17, no. 4, pp. 471-475, 2007.

[8] T. Kaczorek, "A realization problem for positive continuoustime systems with reduced numbers of delays," International Journal of Applied Mathematics and Computer Science, vol. 16, no. 3, pp. 325-331, 2006.

[9] L. Benvenuti, A. D. Santis, and L. Farina, Positive Systems: Theory and Applications, Springer, New York, NY, USA, 2003.

[10] M. A. Rami, F. Tadeo, and A. Benzaouia, "Control of constrained positive discrete systems," in Proceedings of the American Control Conference (ACC '07), pp. 5851-5856, New York, NY, USA, July 2007.

[11] F. Knorn, O. Mason, and R. Shorten, "On linear co-positive Lyapunov functions for sets of linear positive systems," Automatica, vol. 45, no. 8, pp. 1943-1947, 2009.

[12] S. Li, Z. Xiang, and H. R. Karimi, "Stability and $L_{1}$-gain controller design for positive switched systems with mixed timevarying delays," Applied Mathematics and Computation, vol. 222, pp. 507-518, 2013.

[13] H. Liu, P. Shi, H. R. Karimi, and M. Chadli, "Finite-time stability and stabilisation for a class of nonlinear systems with timevarying delay," International Journal of Systems Science, 2014.

[14] H. R. Karimi and H. Gao, "New delay-dependent exponential $H_{\infty}$ synchronization for uncertain neural networks with mixed time delays," IEEE Transactions on Systems, Man, and Cybernetics, Part B: Cybernetics, vol. 40, no. 1, pp. 173-185, 2010.

[15] F. Zhang and Y. Zhang, "State estimation of neural networks with both time-varying delays and norm-bounded parameter uncertainties via a delay decomposition approach," Communications in Nonlinear Science and Numerical Simulation, vol. 18, no. 12, pp. 3517-3529, 2013.

[16] Z. C. Qin, S. Zhong, and J. Q. Sun, "Sliding mode control experiments of uncertain dynamical systems with time delay," Communications in Nonlinear Science and Numerical Simulation, vol. 18, no. 12, pp. 3558-3566, 2013.

[17] M. S. Mahmoud and P. Shi, "Robust stability, stabilization and $H_{\infty}$ control of time-delay systems with Markovian jump parameters," International Journal of Robust and Nonlinear Control, vol. 13, no. 8, pp. 755-784, 2003.
[18] Y. Sun, "Delay-independent stability of switched linear systems with unbounded time-varying delays," Abstract and Applied Analysis, vol. 2012, Article ID 560897, 11 pages, 2012.

[19] M. Xiang and Z. Xiang, "Stability, $L_{1}$-gain and control synthesis for positive switched systems with time-varying delay," Nonlinear Analysis: Hybrid Systems, vol. 9, no. 1, pp. 9-17, 2013.

[20] X. Liu and C. Dang, "Stability analysis of positive switched linear systems with delays," IEEE Transactions on Automatic Control, vol. 56, no. 7, pp. 1684-1690, 2011.

[21] X. Zhao, L. Zhang, and P. Shi, "Stability of a class of switched positive linear time-delay systems," International Journal of Robust and Nonlinear Control, vol. 23, no. 5, pp. 578-589, 2013.

[22] C. Briat, "Robust stability and stabilization of uncertain linear positive systems via integral linear constraints: $L_{1}$-gain and $L_{\infty}$-gain characterization," International Journal of Robust and Nonlinear Control, vol. 23, no. 17, pp. 1932-1954, 2013.

[23] M. Xiang and Z. Xiang, "Observer design of switched positive systems with time-varying delays," Circuits, Systems, and Signal Processing, vol. 32, no. 5, pp. 2171-2184, 2013.

[24] M. Xiang and Z. Xiang, "Finite-time $L_{1}$ control for positive switched linear systems with time-varying delay," Communications in Nonlinear Science and Numerical Simulation, vol. 18, no. 11, pp. 3158-3166, 2013.

[25] Z. Duan, H. R. Karimi, and Z. Xiang, "Stability and $l_{1}$-gain analysis for positive 2D systems with state delays in the Roesser model," Mathematical Problems in Engineering, vol. 2013, Article ID 169713, 10 pages, 2013.

[26] S. Li, Z. Xiang, and H. R. Karimi, "Finite-time $L_{1}$-gain control for positive switched systems with time-varying delay via delta operator approach," Abstract and Applied Analysis, vol. 2014, Article ID 872158, 11 pages, 2014.

[27] S. Li, Z. Xiang, and H. R. Karimi, "Positive $L_{1}$ observer design for positive switched systems," Circuits, Systems, and Signal Processing, vol. 33, no. 7, pp. 2085-2106, 2014.

[28] Z. Duan, Z. Xiang, and H. R. Karimi, "Delay-dependent exponential stabilization of positive 2D switched state-delayed systems in the Roesser model," Information Sciences, vol. 272, pp. 173-184, 2014.

[29] X. Zhang, M. Wang, and J. Zhao, "Stability analysis and antiwindup design of uncertain discrete-time switched linear systems subject to actuator saturation," Journal of Control Theory and Applications, vol. 10, no. 3, pp. 325-331, 2012.

[30] D. Saifia, M. Chadli, H. R. Karimi, and S. Labiod, "Fuzzy control for Electric Power Steering SYStem with assist motor current input constraints," Journal of the Franklin Institute: Engineering and Applied Mathematics, vol. 352, no. 2, pp. 562-576, 2015.

[31] S. Oucheriah, "Global stabilization of a class of linear continuous time-delay systems with saturating controls," IEEE Transactions on Circuits and Systems I: Fundamental Theory and Applications, vol. 43, no. 12, pp. 1012-1015, 1996.

[32] S. Tarbouriech and J. M. G. da Silva Jr., "Synthesis of controllers for continuous-time delay systems with saturating controls via LMI's," IEEE Transactions on Automatic Control, vol. 45, no. 1, pp. 105-111, 2000.

[33] Y.-Y. Cao, Z. Lin, and T. Hu, "Stability analysis of linear timedelay systems subject to input saturation," IEEE Transactions on Circuits and Systems I: Fundamental Theory and Applications, vol. 49, no. 2, pp. 233-240, 2002.

[34] S. Oucheriah, "Synthesis of controllers for time-delay systems subject to actuator saturation and disturbance," Transactions of the ASME-Journal of Dynamic Systems, Measurement and Control, vol. 125, no. 2, pp. 244-249, 2003. 
[35] E. Tissir and A. Hmamed, "Further results on the stabilization of time delay systems containing saturating actuators," International Journal of Systems Science, vol. 23, no. 4, pp. 615-622, 1992.

[36] H. Fang, Z. Lin, and T. Hu, "Analysis of linear systems in the presence of actuator saturation and $L_{2}$-disturbances," Automatica, vol. 40, no. 7, pp. 1229-1238, 2004.

[37] Q. Zheng and F. Wu, "Output feedback control of saturated discrete-time linear systems using parameter-dependent Lyapunov functions," Systems \& Control Letters, vol. 57, no. 11, pp. 896-903, 2008.

[38] Y.-Y. Cao, Z. Lin, and D. G. Ward, "An antiwindup approach to enlarging domain of attraction for linear systems subject to actuator saturation," IEEE Transactions on Automatic Control, vol. 47, no. 1, pp. 140-145, 2002.

[39] T. Hu, Z. Lin, and B. M. Chen, "Analysis and design for discretetime linear systems subject to actuator saturation," Systems \& Control Letters, vol. 45, no. 2, pp. 97-112, 2002.

[40] J. M. Gomes da Silva Jr. and S. Tarbouriech, "Anti-windup design with guaranteed regions of stability for discrete-time linear systems," Systems \& Control Letters, vol. 55, no. 3, pp. 184192, 2006.

[41] D. Saifia, M. Chadli, S. Labiod, and T. M. Guerra, "Robust $H_{\infty}$ static output feedback stabilization of T-S fuzzy systems subject to actuator saturation," International Journal of Control, Automation and Systems, vol. 10, no. 3, pp. 613-622, 2012.

[42] J. Gomes da Silva and S. Tarbouriech, "Antiwindup design with guaranteed regions of stability: an LMI-based approach," IEEE Transactions on Automatic Control, vol. 50, no. 1, pp. 106-111, 2005.

[43] J. Zhang, Y. Wu, Y. Wang, and Z. Gao, "Stability analysis and controller design of positive linear systems subject to actuator saturation," in Proceedings of the 32nd Chinese Control Conference (CCC '13), pp. 33-38, Xi'an, China, July 2013.

[44] J.-S. Zhang, Z. Deng, and Y.-W. Wang, "Robust stability and stabilization of positive interval systems subject to actuator saturation," Asian Journal of Control, vol. 16, no. 5, pp. 1553-1560, 2014.

[45] O. Mason and R. Shorten, "On linear copositive Lyapunov functions and the stability of switched positive linear systems," IEEE Transactions on Automatic Control, vol. 52, no. 7, pp.13461349, 2007.

[46] T. Hu, Z. Lin, and B. M. Chen, "An analysis and design method for linear systems subject to actuator saturation and disturbance," Automatica, vol. 38, no. 2, pp. 351-359, 2002. 


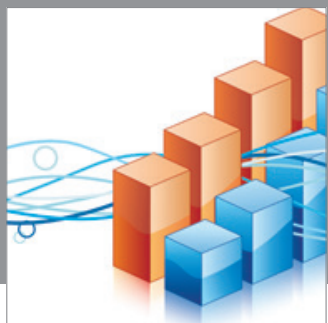

Advances in

Operations Research

mansans

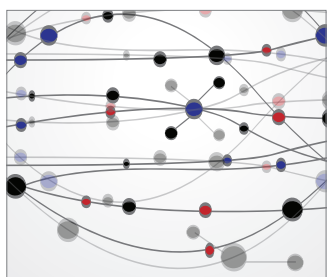

The Scientific World Journal
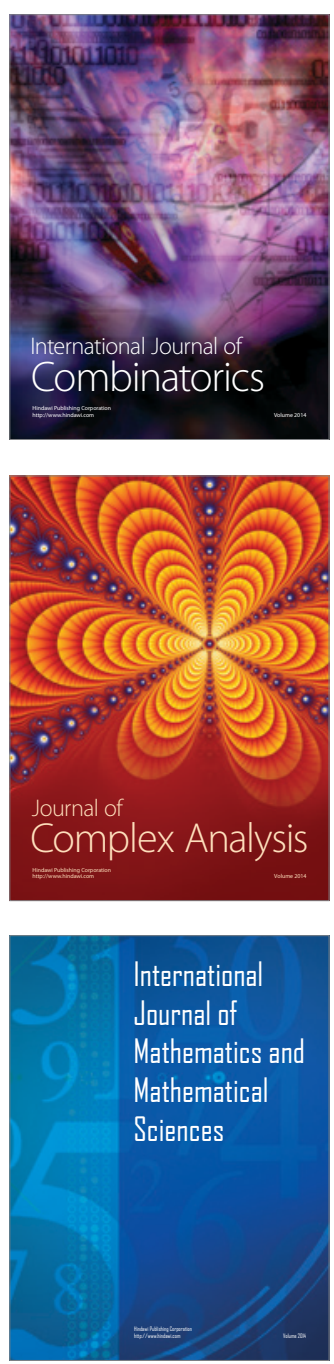
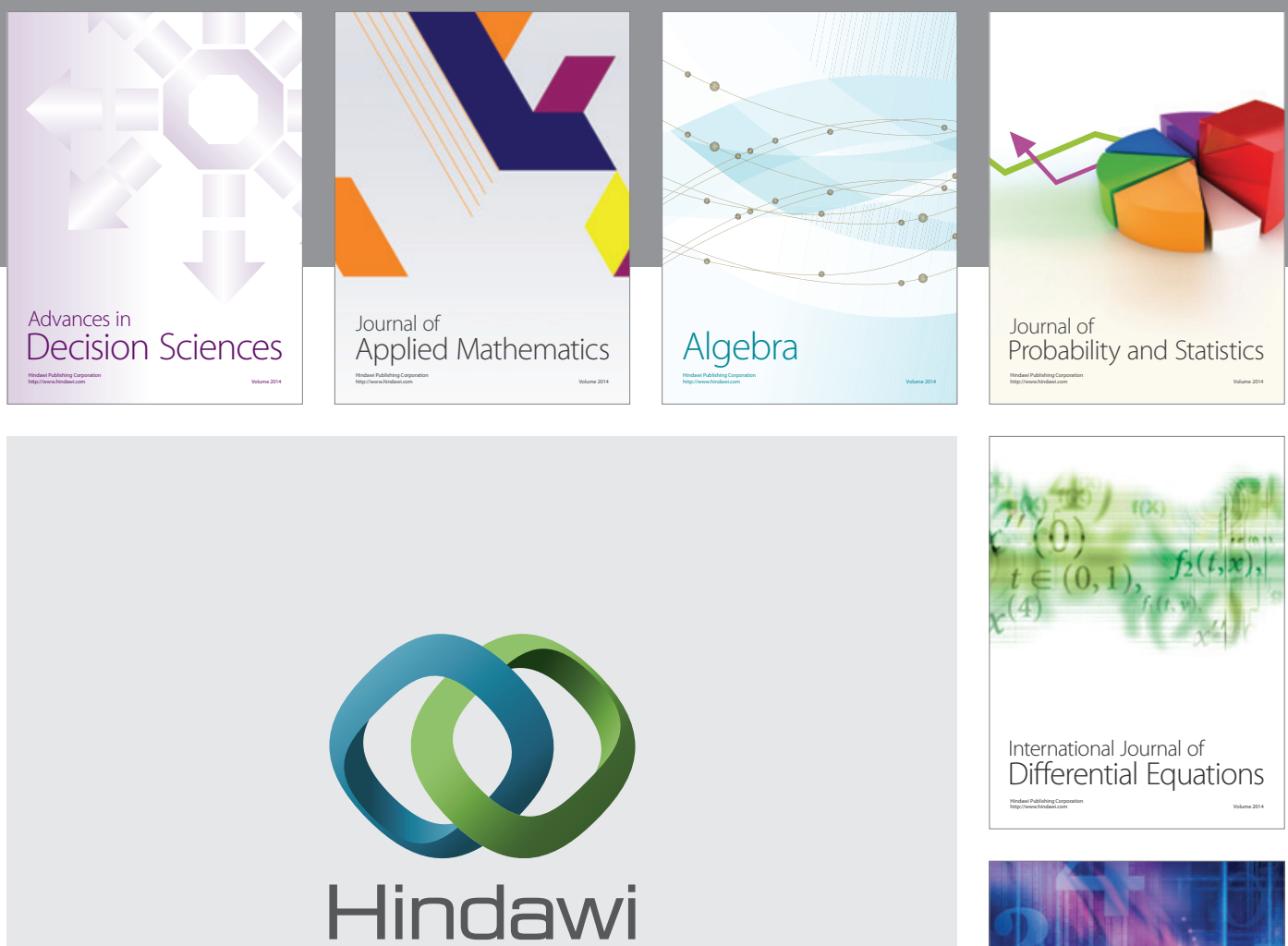

Submit your manuscripts at http://www.hindawi.com
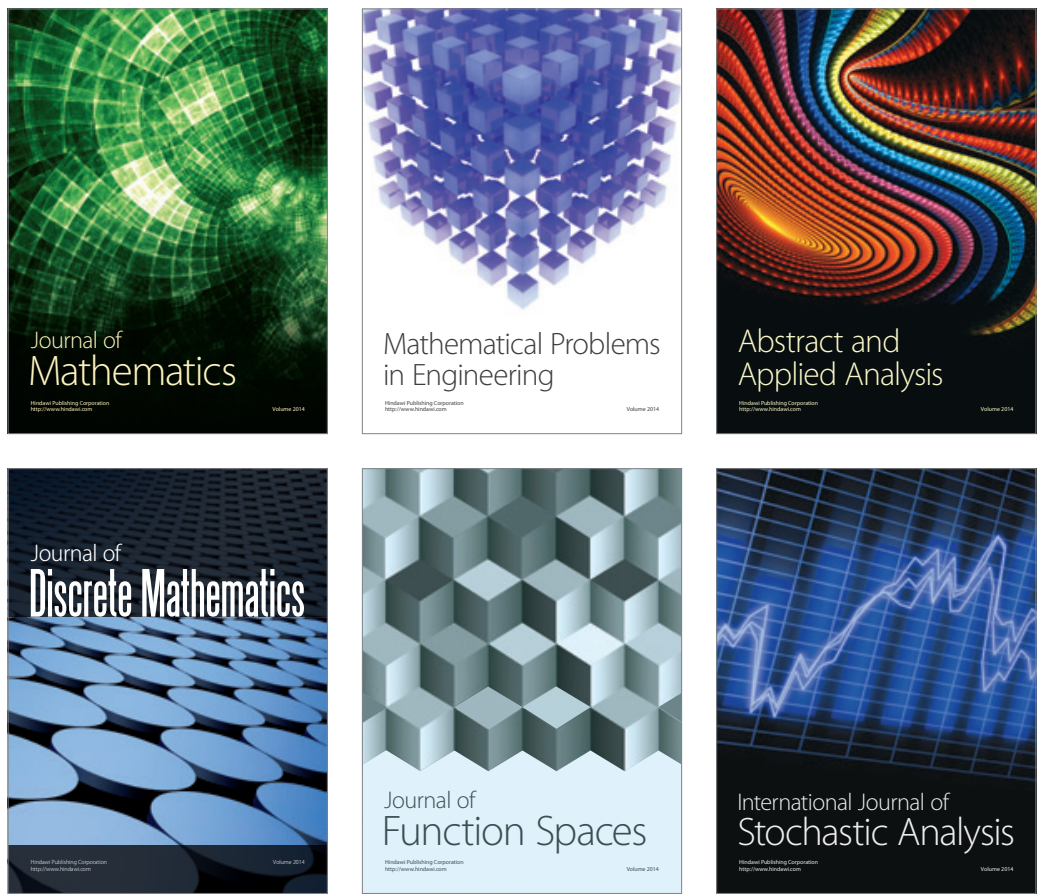

Journal of

Function Spaces

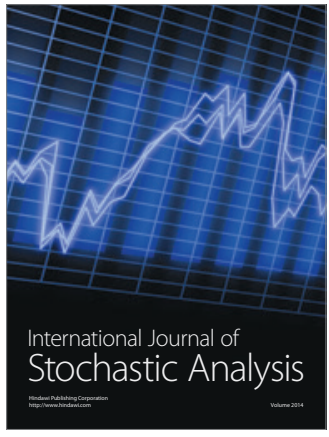

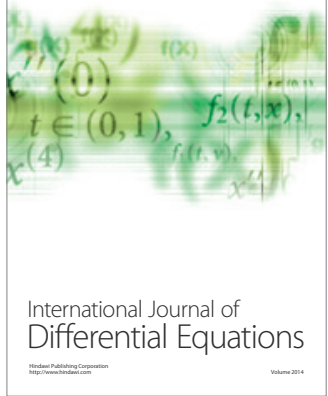
